\title{
FOMENTO DA PESQUISA CIENTÍFICA NACIONAL: O AUXÍLIO FINANCEIRO AO PESQUISADOR NA CHAMADA UNIVERSAL
}

\author{
Marcos César Chaves da Fonseca* \\ Ricardo Barros Sampaio** \\ Angela Terezinha de Souza Wyse ${ }^{* * *}$
}

Resumo: No modelo de desenvolvimento científico nacional, fração expressiva dos projetos de pesquisa são fomentados por chamadas públicas, que estabelecem os critérios para a seleção das propostas de maior relevância e de mérito científico. A Chamada Pública Universal do Conselho Nacional de Desenvolvimento Científico e Tecnológico ( $\mathrm{CNPq}$ ) apoiou, no período de 2005 a 2016, diferentes instituições, precisamente 569, distribuídas em todo o território nacional. Dessas instituições, 52\% eram Instituições de Ensino Superior (IES) e 40\% delas possuíam Programas de Pós-Graduação (PPG). Grande parte dos recursos apoiados, cerca de $86 \%$, foram para as grandes áreas tradicionais do conhecimento e para a compra de bens e equipamentos. A produção do conhecimento dessas instituições demonstrou sensibilidade aos grandes temas nacionais, como no caso do surto do zika vírus, de 2015, ocasião em que foram submetidos 179 trabalhos sobre o tema. Contudo, o modelo de fomento necessita ser aperfeiçoado e adequar-se às demandas dos núcleos produtores do conhecimento e da sociedade.

Palavras-chaves: Fomento. Programa de Pós-Graduação. Promoção de pesquisa científica.

\section{Introdução}

O Brasil ocupou o $13^{\circ}$ lugar na produção de conhecimento indexado nas bases de dados científicos internacionais no período de 2011 a 2016 (CLARIVATE ANALYTICS, 2016), demonstrando um crescimento regular ao longo dos anos. A maior parte da produção desse conhecimento foi proporcionada por recursos públicos aplicados diretamente no sistema educacional brasileiro para a capacitação de recursos humanos ou fomento de projetos de

\footnotetext{
* Doutorando do Programa de Pós-Graduação em Educação em Ciências: Químicas da Vida e Saúde (PPGEC) da Universidade Federal do Rio Grande do Sul - UFRGS. E-mail: marchafon@ gmail.com.

** Professor Doutor colaborador do Programa de Pós-Graduação em Ciência da Informação (PPGCINF) da Universidade de Brasília - UnB. E-mail: rsampaio.br@gmail.com.

${ }^{* * *}$ Professora Doutora Titular de Bioquímica (ICBS) da Universidade Federal do Rio Grande do Sul - UFRGS. Email:wyse@ufrgs.br.
} 
pesquisa desenvolvidos, na sua maioria, por universidades públicas e por centros de pesquisa nacionais.

Esse crescimento é atribuído a uma sequência de decisões importantes que o país tomou na segunda metade do século passado, quando priorizou a instalação dos programas de pósgraduação nos centros de excelência do ensino superior (GTRU, 1968). Também contribuíram as sucessivas diretrizes, estratégias e metas definidas no âmbito do Sistema Nacional de PósGraduação (SNPG) e dos Planos Nacionais de Pós-Graduação (PNPG), que trouxeram ao longo dos anos uma evolução nos níveis de produção acadêmica, aumentando, como isso, a qualidade dos programas/pesquisas (CAPES, 2010).

O resultado foi a centralização do financiamento público nas Instituições de Ensino Superior (IES), tendo os Programas de Pós-Graduação (PPG) como grandes promotores do conhecimento e da formação de recursos humanos associados à pesquisa, com destaque para as áreas tradicionais do conhecimento científico, em regiões com boa infraestrutura, o que é derivado do processo de desenvolvimento nacional da ciência e da tecnologia (SANTOS, 2015).

É razoável declarar que esse processo de centralização teve um êxito considerável, pois colocou a pós-graduação no centro da pesquisa científica. No entanto, faz-se necessário somar a esses esforços o financiamento público à pesquisa direta, que aqui se traduz como auxílio financeiro ao pesquisador. Os auxílios são destinados ao desenvolvimento de estudos e pesquisas de natureza científica realizados por pessoas físicas na condição de estudantes ou de pesquisadores com doutorado. Essas despesas indicam os objetos de investimento que correspondem aos esforços da manutenção da pesquisa no Brasil (BRASIL, 2013).

Considerando que há poucos estudos relacionando os instrumentos de fomento e os investimentos realizados em ciência tecnologia e inovação, o objetivo deste trabalho é analisar o investimento efetuado pela Chamada Universal do CNPq no período de 11 anos (2005 a 2016), que compreendem os projetos implementados nas edições das Chamadas de 2004, 2006, 2007, 2008, 2009, 2010, 2011, 2012, 2013, 2014 e 2016. Focamos, assim, na participação dos PPGs, com ênfase na área das Ciências da Saúde, bem como na concentração das grandes áreas do conhecimento do CNPq. Buscou-se, igualmente, retratar as características das unidades da 


\section{\#tear}

Federação e as IES pelo acumulado das categorias de despesa, de custeio ou de capital, que poderiam ajudar a entender a evolução da produção científica em seu processo de desenvolvimento e discutir o perfil do investimento realizado diretamente ao pesquisador no ambiente das IES e, por consequência, dos seus PPGs.

Definiu-se como ponto centro de análise a Chamada Pública Universal do CNPq, que se configura como instrumento de apoio e estímulo à pesquisa científica no elemento despesa do auxílio financeiro ao pesquisador, na natureza de custeio e de capital. Entende-se como custeio as despesas que não contribuem diretamente para a formação ou a aquisição de bens patrimoniáveis e como capital as despesas destinadas à aquisição do bem patrimoniável (BRASIL, 2013). A Chamada representa o modelo de fomento não induzido, apoiando pesquisas em todas as áreas do conhecimento. Esse instrumento de fomento busca contribuir significativamente para o desenvolvimento científico, tecnológico e de inovação no país, propondo-se a apoiar pesquisadores em distintas faixas de desenvolvimento e maturidade acadêmica, bem como em diferentes níveis de infraestrutura de operação.

Desde 2007, essa chamada divide o auxílio em níveis de concorrência, em outras palavras, em faixas de financiamento crescente representadas pelas letras A, B e C e em critérios de senioridade acadêmica. Portanto, nossa hipótese é que o exame dessa Chamada pode encontrar aspectos significativos do sistema de fomento nacional, que é um indutor da produção científica. Essa investigação pode auxiliar os gestores do sistema a direcionarem ações e políticas para que se atentem ao princípio da isonomia, à distribuição de recursos e, consequentemente, a um desenvolvimento científico mais equânime no país. Pode, ainda, auxiliar na avaliação da aplicabilidade do conhecimento científico gerado, baseando-se nos artigos científicos publicados e no trabalho dos grandes centros de pesquisa. Além disso, é relevante entender se a distribuição dos recursos está comprometida em solucionar as questões e os problemas que permeiam a sociedade, como foi o caso do zika vírus. 


\section{Metodologia}

Para o desenvolvimento deste estudo foram utilizadas a análise de dados descritiva e a análise bibliográfica no intuito de observar o perfil da distribuição do auxílio ao pesquisador no fomento oferecido pela Chamada Pública Universal, no período de 2005 a 2016. Ademais, a relação das grandes áreas do conhecimento, em especial a área do conhecimento das Ciências da Saúde com os Programas de Pós-Graduação. Esses métodos permitiram a análise dos recursos distribuídos pela Chamada, bem como permitiram que fossem comparados ao observado nos dados bibliográficos referentes à produção científica dos PPGs e informações constantes na página eletrônica da Coordenação de Aperfeiçoamento de Pessoal de Nível Superior (CAPES).

A análise de dados descritiva, segundo Sampaio, Assunção e Fonseca (2018), tem por finalidade a observação do fenômeno estatístico em que a coleta, a organização e a apresentação dos dados obtidos são analisadas. Essa análise permitiu organizar os dados do fomento contidos nas tabelas do sistema gerenciador de banco de dados Oracle do CNPq e apresentá-los em formato visual utilizando o software livre Rstudio e gráficos de dispersão com tamanho de ponto variável.

O Rstudio é um ambiente de desenvolvimento IDE (Integrated Development Environment) para a linguagem de programação R que inclui um console, um editor de realce de sintaxe que suporta a execução direta de código, bem como ferramentas para plotagem, histórico, depuração e gerenciamento de espaço de trabalho (FOAS, 2018) . Além do Rstudio, foram utilizados vários pacotes de códigos estruturados para execução de tarefas no ambiente, dentre eles o pacote ggplot2 para visualização de dados, que divide gráficos em componentes semânticos como escalas e camadas (WILKINSON, 2007)

Dentre as informações analisadas, o gráfico de bolhas foi o que melhor representou os dados deste estudo. Trata-se de uma variação do gráfico de dispersão, em que uma terceira dimensão é incorporada por um valor numérico adicional, representado pelo tamanho dos pontos. Ele é formado por três variáveis numéricas com entrada, eixo X, eixo Y e uma pelo tamanho do ponto do gráfico (HOLTZ; HEALY, 2018). Os dados foram coordenados pelas informações de capital, eixo $\mathrm{X}$ e dados de custeio, eixo $\mathrm{Y}$, das propostas reconhecidas como meritórias no 
processo de avaliação do CNPq. O tamanho do ponto variou no Gráfico 1, como veremos, em razão do volume de recursos que cada grande área de conhecimento recebeu; no Gráfico 2 variou devido ao volume de recursos que cada área do conhecimento recebeu; e no Gráfico 3 variou: a) pelo volume de recursos que cada unidade da Federação recebeu e b) pelo volume que cada instituição recebeu no período do estudo.

As análises do fomento foram discutidas ao longo do artigo em função dos achados da análise bibliográfica. A seleção dos dados bibliográficos envolveu uma base preliminar com artigos que tratavam da produção científica (TREITA; FILHO; RABELO, 2013) dos PPGs, visando confirmar o estado da arte no tema, com filtro para a área das Ciências da Saúde e dos documentos de avaliação dos PNPGs, bem como os painéis da ferramenta de georreferenciamento, Geocaps, e indicadores de avaliação dos programas da CAPES.

\section{Resultados e discussão}

Os dados mostraram que a Chamada Universal contemplou 51.270 projetos em 569 instituições no período de 2005 a 2016, sendo que 51\% delas eram IES, e, dentre elas, 40\% eram instituições de ensino superior que possuíam PPG no período analisado, conforme comparativo com os dados da distribuição dos Programas de Pós-graduação no Brasil por IES do Sistema de Informações Georreferenciadas (GEOCAPS, 2018), o que retrata o perfil da produção científica brasileira. Esse dado assevera-se quando o indicador de classificação de instituições acadêmicas e de pesquisa do SCImago Journal \& Country Rank (SCIMAGO, 2018) mostra que 86\% das instituições classificadas no ranking das instituições brasileiras são IES e que 97\% das instituições classificadas possuem programas de pós-graduação stricto sensu.

Essa verificação demonstra o êxito da produção científica nacional, a qual está concentrada nas instituições públicas de ensino superior. Esse dado pode ser um indicador de assertividade dos investimentos realizados pela Chamada Universal no período estudado, pois todas as instituições relacionadas no ranking de classificação de instituições acadêmicas e de pesquisa do SCImago Journal \& Country Rank tiveram projetos apoiados no âmbito da Chamada, sendo que as dez melhores instituições receberam vinte e nove vezes mais 
investimentos no período oriundo da Chamada do que as dez instituições com menor índice de classificação no referido ranking. Acresce-se que as instituições de melhor desempenho estão situadas nas regiões sudeste e sul, as quais possuem infraestrutura e grupos de pesquisa bem consolidados, dividindo-se em IES e instituições de governo ligadas à saúde e CT\&I.

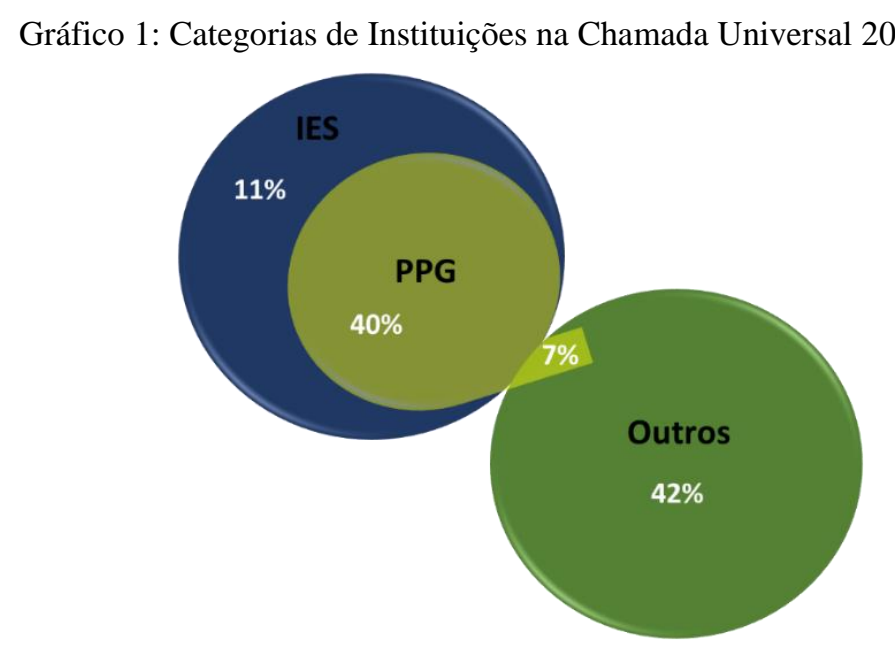

Fonte: Elaboração dos autores

No contexto histórico da Chamada, as IES captaram grande parte dos recursos distribuídos e os concentraram nas grandes áreas do conhecimento, sendo em sua maioria divididos nas áreas de Ciências Biológicas, Ciências Exatas e da Terra, Engenharias, Ciências Agrárias e Ciências da Saúde, representadas pelo tamanho do ponto e pela região em azul do Gráfico 1. Essas áreas captaram a maior quantidade de recursos acumulados no período estudado - cerca de $85,9 \%$ do volume total disponibilizado e em proporções semelhantes que variaram de $19,3 \%, 18,4 \%, 17,2 \%, 16,3 \%$ e 14,6\%, respectivamente, demonstrando que, nos moldes da modalidade de fomento, houve maior atração para as ciências experimentais e por fomento de natureza de capital. 
Gráfico 2: Distribuição do auxílio à pesquisa na Chamada Universal por área

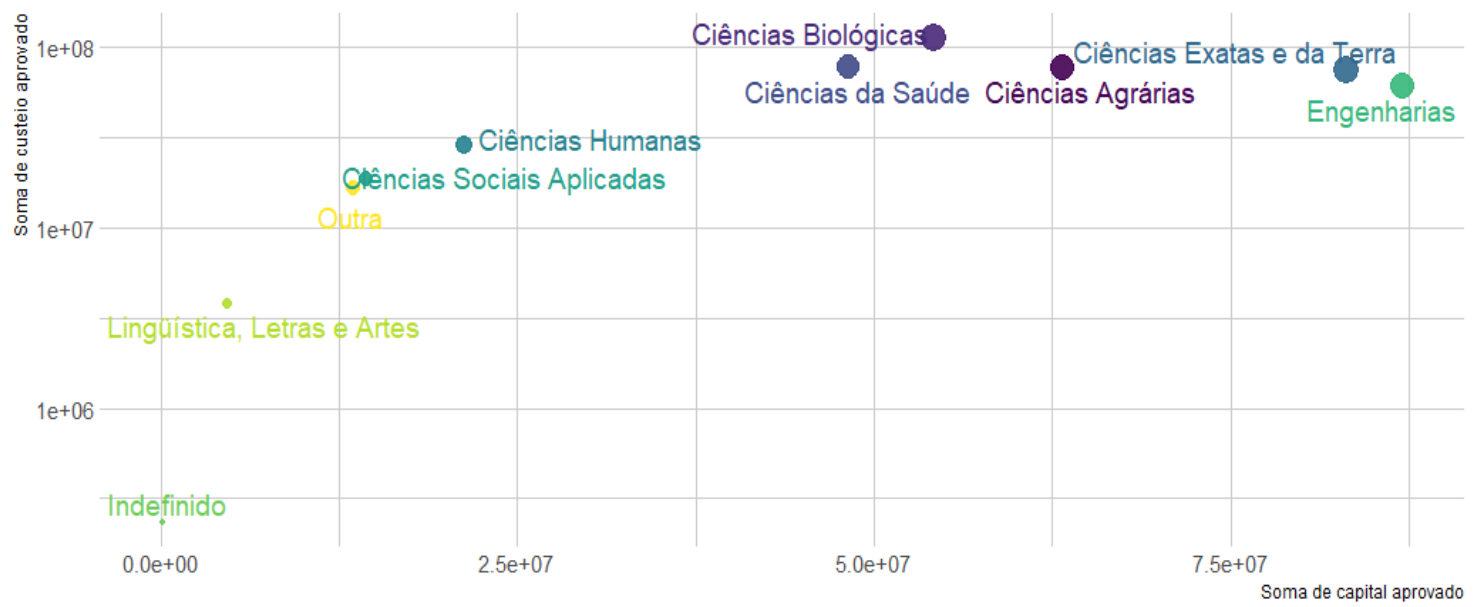

Fonte: elaboração dos autores

A média do valor fomentado por projeto nessas grandes áreas do conhecimento variou de $\mathrm{R} \$ 9.782,58$ para a faixa $A, R \$ 22.668,70$ para a faixa $B$ e $R \$ 52.884,39$ para a faixa $C$, o que corresponde a um valor médio geral para a modalidade de fomento de R $\$ 27.392,08$ para um período de dois a três anos a depender da edição da Chamada.

Traçando um paralelo entre os PPGs da área das Ciências da Saúde e sua adesão à modalidade de financiamento oferecida pela Chamada Universal do CNPq, observou-se que, apesar de a grande área ocupar a segunda colocação em número de PPGs - com 681 unidades - e de a maioria deles - $57 \%$ do total - possuir unidades com os dois níveis do stricto senso — mestrado e doutorado - a grande área do conhecimento performou com $15 \%$ do acumulado de projetos aprovados na Chamada Universal e somente na quinta colocação no volume acumulado de recursos distribuídos pela modalidade de fomento (GEOCAPS, 2018).

A adesão dos projetos das áreas do conhecimento representou a proporção acumulada de 29\% para Medicina, 15\% para Farmácia, 12\% para Odontologia, 11\% para Enfermagem, 10\% para Saúde Coletiva, 8\% para Fisioterapia, 7\% para Educação Física, 6\% para Nutrição e 3\% 
para Fonoaudiologia. Nota-se que os projetos aprovados mantiveram seus auxílios nas rubricas de capital e custeio próximos ao original solicitado.

Gráfico 3: Distribuição do fomento entre áreas do conhecimento das Ciências da Saúde.

a) Custeio Solicitado

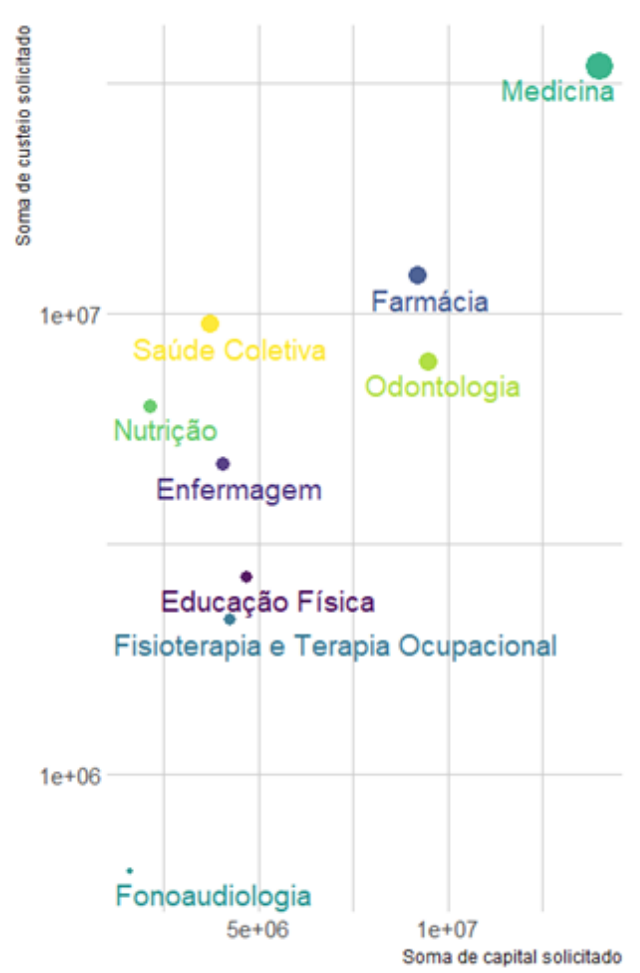

b) Custeio Aprovado

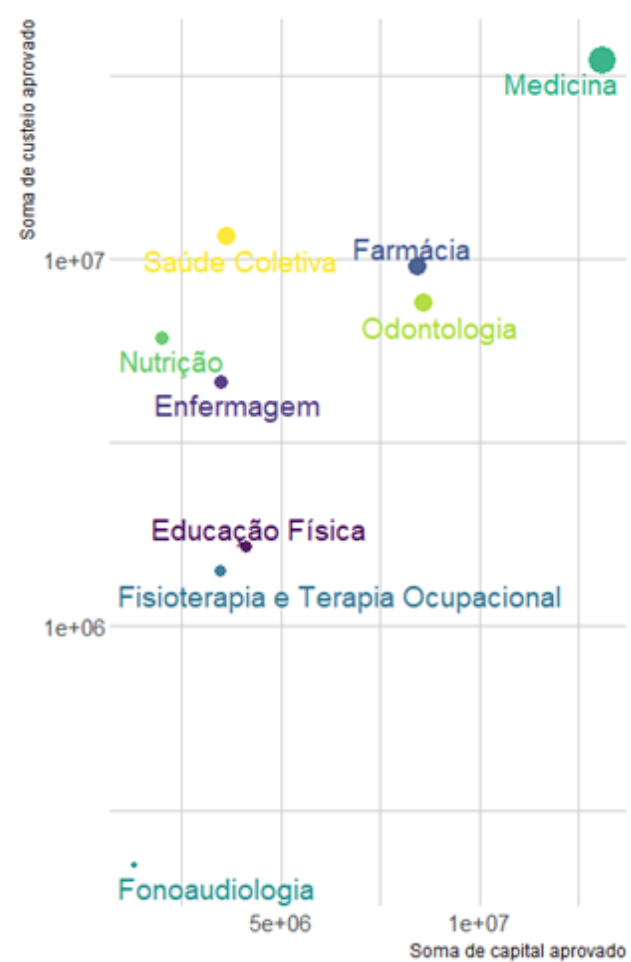

Fonte: elaboração dos autores

O Gráfico 3 (a e b) remete ao comparativo do acumulado de recursos solicitados e aprovados para financiamento dos projetos na Área das Ciências da Saúde na Chamada Universal. Especificamente, no Gráfico 3b, pode-se observar a distribuição do auxílio ante as características das áreas pelo volume de recursos aprovados dentro dos recursos disponíveis e efetivamente recebidos pelos projetos de pesquisa. A relação demonstra que as áreas demandaram quantidade de recursos diferentes em volume, natureza e na relação entre custeio e capital. 
Essa característica apresentada por algumas áreas pode ensejar um perfil de projeto e tema discutido para aquele fomento, ou até mesmo expressar a necessidade da IES na qual o PPG está inserido, bem como o nível de maturidade de seus integrantes, pois algumas áreas consumiram quantidades maiores de fomento na rubrica de capital e outras na rubrica de custeio.

Como exemplo, pode-se citar a área de Fonoaudiologia, com PPGs centralizados nas Regiões Sudeste e Sul (CAPES, 2017), que possuem acumulado de projetos na faixa A, 69\% do total, seguidas de $23 \%$ para a faixa $\mathrm{B}$ e $7 \%$ para a faixa $\mathrm{C}$, situação que evidencia projetos de baixo custo e com demandas na rubrica de custeio. A área da Medicina, cujos PPGs se concentram em sua maioria na Região Sudeste (CAPES, 2017), possui médias acumuladas equilibradas nas faixas da Chamada Universal - faixa A, 40\%; faixa B, 32\%; e faixa C, 28\%.Pode-se inferir, nesse sentido, que se tratam de projetos robustos em vários níveis de complexidade, que variaram na faixa em valores de $\mathrm{R} \$ 30$ mil e $\mathrm{R} \$ 150$ mil reais.

Ademais, a área de Medicina é representada mais fortemente no Estado de São Paulo, mais especificamente, na Universidade de São Paulo, USP. Esse fato também foi observado por Lievore, Picinin e Pilatti (2017) para os PPGs da especialidade de cirurgia-geral da Medicina III, no período de 1995 a 2014, de acordo com os relatórios dos PNPGs.

Gráfico 4: Distribuição do fomento entre áreas do conhecimento das Ciências da Saúde.
a) Distribuição do fomento por UF
b) Distribuição do fomento por instituições
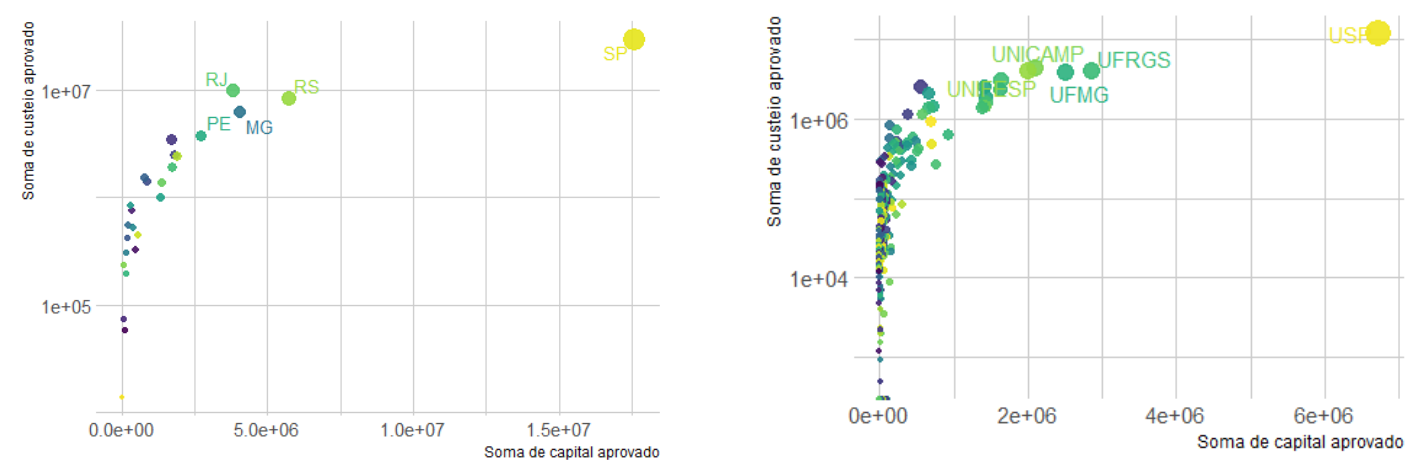

Fonte: elaboração dos autores 
As áreas das Ciências da Saúde receberam maior auxílio acumulado no período para as unidades da Federação e para instituições mais tradicionais do cenário nacional de ciência e tecnologia. Esse fato também foi observado nas unidades dos PPGs dessas áreas, as quais também possuem conceitos relevantes nas avaliações da CAPES. Isso significa que a Chamada está tendo um apoio efetivo, pois fomenta projetos de mérito reconhecido em instituições bem avaliadas e com programas de pós-graduação estruturados, colaborando com o desenvolvimento científico nacional.

Esse sistema de produção do conhecimento nacional demonstrou capacidade de articulação e de resposta no surto do zika vírus em 2015, quando se constatou a mobilização dos PPGs com a submissão de 179 trabalhos contendo a palavra zika no seu título ecom o apoio a 47 trabalhos na edição de 2016 da Chamada. Essa mobilização também foi verificada por Albuquerque et al. (2017), que demonstraram o crescimento de trabalhos indexados no Scopus e no Web of Science entre 2014 e 2016 de autores brasileiros. O crescimento chegou a 1600 trabalhos, aproximadamente. O Brasil ficou em segundo lugar no número de publicações relevantes, isso contribui para o conhecimento do problema, controle da doença e possíveis caminhos de tratamento dos casos de microcefalia.

\section{Considerações gerais}

Neste estudo, observa-se que o acumulado do fomento recebido pelos PPGs retrata as características da produção científica brasileira, que é focada na produção de artigos, que, por sua vez, constituem ponto central nos métodos de avaliação da pós-graduação brasileira por meio do investimento em CT\&I das agências de fomento nacionais. Esse foco define todo o sistema da formação, produção e desenvolvimento da massa crítica nacional.

A comparação feita neste estudo entre a análise descritiva realizada e os achados na literatura demonstram que o modelo de fomento da Chamada Universal não impõe barreiras de temáticas ou de requisitos específicos. A única exigência é o título de doutor, etapa inicial da longa carreira de um pesquisador no Brasil. Em contrapartida, existem enquadramentos para os limites de recursos, situação que condiciona e, ao mesmo tempo, salvaguarda os pesquisadores 
em início de carreira e egressos dos PPGs, que buscam estabilidade nesse segmento muitas vezes extremamente competitivo.

O estudo também demonstrou a lógica de fomento em que um grande número de IES é atendido, mas com valor de recursos limitados, situação que tem como consequência a pulverização dos recursos diante da escassez de fomento. Essa lógica pode colocar em xeque a própria modalidade de fomento, pois se torna difícil o dimensionamento de seus impactos nas áreas do conhecimento. Isso foi observado, especialmente, nas grandes áreas das Ciências da Saúde, que se tornam cada vez mais interdisciplinares e necessitadas de apoio contínuo de longo prazo para a produção de conhecimento e de propostas de solução para os grandes temas comprometedores da saúde da população.

Nas áreas do conhecimento da grande área das Ciências da Saúde, ficou latente a diferença entre as áreas que possuem maior histórico de desenvolvimento e consolidação na distribuição de fomento e as áreas com desenvolvimento de menor histórico. Em relação a essa característica, a centralização do volume de recursos demonstrada pelas IES do Estado de São Paulo, especificamente a USP representa a análise do valor acumulado. Contudo, tanto as áreas com maior histórico de desenvolvimento quanto as de menor denotam fatores heterogêneos que devem ser considerados e calibrados na promoção da pesquisa científica nacional.

É possível presumir que os modelos de fomento tradicionais estão tendo dificuldades para se encaixar nos novos desafios trazidos pelas exigências legais e pela sociedade, pois é importante que se avaliem os impactos das ações e a relação com o avanço do conhecimento produzido, ou seja, a sociedade exige eficiência e transparência dos gastos públicos. Essa dificuldade explicita o modelo de produção científica nacional, observado também nos dados obtidos neste estudo. Ainda temos poucos dados, no entanto, que permitam a determinação da correlação direta entre a produção fomentada e a relevância concreta para a sociedade. Por outro lado, é importante enfatizar que tanto a pesquisa aplicada quanto a básica devem ser motivadas, pois a pesquisa básica é fundamental na geração do conhecimento e na criação de novos métodos e novas tecnologias. No entanto, os resultados e as descobertas obtidos na pesquisa devem ser 
divulgados pelos pesquisadores à sociedade, possibilitando o debate do conhecimento que deve ser incentivado pelas instituições, governo e meios de comunicação (mídia).

As análises (descritiva e bibliográfica) aplicadas aos dados da Chamada Universal de 2005 a 2016 mostraram que o volume acumulado da distribuição do fomento para as IES com PPGs está concentrado nas unidades com melhor classificação nos programas de avaliação da CAPES, demonstrando que o fomento seguiu o tradicional desenvolvimento científico brasileiro e apoiou projetos em todos os estados brasileiros. O acumulado do fomento mostrou destaque para financiamento de equipamentos e bens de capital para as áreas do conhecimento e menos evidência para o apoio a projetos de pesquisa interdisciplinares. A maturidade da equipe de projeto é considerada pela avaliação das comunidades acadêmicas das várias regiões do país, mas pouco identificada na análise em questão em razão da ausência de comparabilidade. Essa disfuncionalidade pode promover distorções na distribuição de recursos e a concentração nas unidades mais desenvolvidas.

\title{
NATIONAL FOSTERING SCIENTIFIC RESEARCH: FINANCIAL SUPPORT TO THE RESEARCHER IN THE UNIVERSAL CALL
}

\begin{abstract}
In the model of national scientific development, the expressive fraction of the research projects are fomented by public calls, which establish the criteria for the selection of proposals of greater relevance and scientific merit. The Universal Public Call of the National Council of Scientific and Technological Development (CNPq) supported, between 2005 and 2016, different institutions, precisely 569, distributed throughout the national territory. Of these institutions, $52 \%$ were Higher Education Institutions (HEI) and $40 \%$ of them had Postgraduate Programs (PPG). A large part of the resources supported, about $86 \%$, went to the large traditional areas of knowledge and to the purchase of goods and equipment. The knowledge production of these institutions showed sensitivity to the major national issues, as in the case of the zika virus outbreak of 2015, when 179 papers were submitted on the subject. However, the model of development needs to be improved and adapted to the demands of the knowledge-producing nuclei and society.
\end{abstract}

Keywords: Fostering. Graduate program. Scientific research promotion. 


\section{Referências}

ALBUQUERQUE, P. C. et al. Bibliometric Indicators of the Zika Outbreak. Plos - Neglected Tropical Diseases, San Francisco, California, US, 19 jan. 2017.

BRASIL. Ministério do Planejamento e Gestão. RESOLUÇÃO CONJUNTA Nº1 DE 17 DE JANEIRO DE 2013, 2013. Disponível em:

<http://www.planejamento.pr.gov.br/arquivos/File/Arquivos\%20PDF\%20/Resolucao_conjunta_ n_01_17_de_janeiro_de_2013.pdf>. Acesso em: 16 out. 2018.

CAPES. Plano Nacional de Pós-Graduação - PNPG 2011-2020. Fundação CAPES, 2010.

Disponível em: <http://www.capes.gov.br/plano-nacional-de-pos-graduacao>. Acesso em: 6 out. 2018.

CAPES. Relatório de avaliação - Educação Física - Avaliação Quadrienal 2017. CAPES.

Brasília, p. 4., 2017.

CAPES. Relatório de avaliação - Medicina 2 - Avaliação Quadrienal 2017. CAPES. Brasília, p. 1., 2017.

CLARIVATE ANALYTICS. CAPES. Research in Brazil, 2016. Disponível em:

<https://www.capes.gov.br/images/stories/download/diversos/17012018-CAPES-InCitesReportFinal.pdf $>$. Acesso em: 15 out. 2018.

CNPQ. CHAMADA UNIVERSAL MCTI/CNPq Nº 01/2016, Brasília, 2016.

FOAS. Foundation for Open Access Statistics. RStudio, 2018. Disponível em:

<https://www.rstudio.com/products/RStudio/>. Acesso em: 13 out. 2018.

GEOCAPS. Geocaps Dados Estatísticos. Geocaps Dados Estatísticos, 2018. Disponível em: <https://geocapes.capes.gov.br/geocapes/>. Acesso em: 6 out. 2018.

GTRU. Grupo de Trabalho da Reforma Universitária. Revista Paz e Terra, Rio de Janeiro, v. 9, p. 243-282, 1968.

HOLTZ, Y.; HEALY, C. From Data to Viz. From Data to Viz, 2018. Disponível em: <https://www.data-to-viz.com/>. Acesso em: 14 out. 2018.

LIEVORE, C.; PICININ, C. T.; PILATTI, L. A. As áreas do conhecimento na pós-graduação stricto sensu brasileira: crescimento longitudinal entre 1995 e 2014. Ensaio: Aval. Pol. Públ. Educ., Rio de Janeiro, v. 25, n. 94, p. 207-237, jan./mar 2017. 
SAMPAIO, N. A. D. S.; ASSUNÇÃO, A. R. P. D.; FONSECA, B. B. D. Estatística Descritiva. Belo Horizonte: Editora Poisson, 2018.

SANTOS, S. M. D. Capes - Teses Premiadas. O desenho das univerisades brasileiras nos rankings internacionais: área de destaque da produção científica brasileira, 2015. Disponível em: <https://www.capes.gov.br/images/stories/download/pct/2016/Teses-Premiadas/CienciasSociais-Aplicadas-Solange-Maria-dos-Santos.PDF>. Acesso em: 15 out. 2018.

SCIMAGO. SCImago Journal \& Country Rank. Scimago Journal \& Country Rank, 2018. Disponível em: <https://www.scimagojr.com/countrysearch.php?country=br>. Acesso em: $1^{\circ}$ out. 2018.

TREITA, F. T.; FILHO, J. R. F. S. A. P.; RABELO, L. M. Metodologia de pesquisa bibliográfica com a utilização. Produção - Scielo, 2013. Disponível em: <http://www.scielo.br/pdf/prod/2013nahead/aop_prod0312.pdf>. Acesso em: 14 out. 2018.

WILKINSON, L. The Grammar of Graphics. Journal of Statistical Software, New York, jan. 2007. Disponível em: <https://www.jstatsoft.org/issue/view/v017>. Acesso em: 14 out. 2018. 